\title{
Dorsal anterior cingulotomy and anterior capsulotomy for severe, refractory obsessive-compulsive disorder: a systematic review of observational studies
}

\author{
Lauren T. Brown, BA, ${ }^{1}$ Charles B. Mikell, MD, ${ }^{1}$ Brett E. Youngerman, MD, ${ }^{1}$ Yuan Zhang, MS, MA, ${ }^{2}$ \\ Guy M. McKhann II, MD, ${ }^{1}$ and Sameer A. Sheth, MD, PhD ${ }^{1}$
}

'Department of Neurological Surgery, Columbia University; and 2Department of Biostatistics, Mailman School of Public Health, Columbia University, New York, New York

OBJECTIVE The object of this study was to perform a systematic review, according to Preferred Reporting Items of Systematic reviews and Meta-Analyses (PRISMA) and Agency for Healthcare Research and Quality (AHRQ) guidelines, of the clinical efficacy and adverse effect profile of dorsal anterior cingulotomy compared with anterior capsulotomy for the treatment of severe, refractory obsessive-compulsive disorder (OCD).

METHODS The authors included studies comparing objective clinical measures before and after cingulotomy or capsulotomy (surgical and radiosurgical) in patients with OCD. Only papers reporting the most current follow-up data for each group of investigators were included. Studies reporting results on patients undergoing one or more procedures other than cingulotomy or capsulotomy were excluded. Case reports and studies with a mean follow-up shorter than 12 months were excluded. Clinical response was defined in terms of a change in the Yale-Brown Obsessive Compulsive Scale (Y-BOCS) score. The authors searched MEDLINE, PubMed, PsycINFO, Scopus, and Web of Knowledge through October 2013. English and non-English articles and abstracts were reviewed.

RESULTS Ten studies involving 193 participants evaluated the length of follow-up, change in the Y-BOCS score, and postoperative adverse events (AEs) after cingulotomy ( $n=2$ studies, $n=81$ participants) or capsulotomy ( $n=8$ studies, $n=112$ participants). The average time to the last follow-up was 47 months for cingulotomy and 60 months for capsulotomy. The mean reduction in the Y-BOCS score at 12 months' follow-up was 37\% for cingulotomy and $55 \%$ for capsulotomy. At the last follow-up, the mean reduction in Y-BOCS score was $37 \%$ for cingulotomy and $57 \%$ for capsulotomy. The average full response rate to cingulotomy at the last follow-up was $41 \%$ (range $38 \%-47 \%, n=2$ studies, $n=51$ participants), and to capsulotomy was $54 \%$ (range $37 \%-80 \%, n=5$ studies, $n=50$ participants). The rate of transient AEs was $14.3 \%$ across cingulotomy studies ( $n=116$ procedures) and $56.2 \%$ across capsulotomy studies $(n=112$ procedures). The rate of serious or permanent AEs was $5.2 \%$ across cingulotomy studies and $21.4 \%$ across capsulotomy studies.

CONCLUSIONS This systematic review of the literature supports the efficacy of both dorsal anterior cingulotomy and anterior capsulotomy in this highly treatment-refractory population. The observational nature of available data limits the ability to directly compare these procedures. Controlled or head-to-head studies are necessary to identify differences in efficacy or AEs and may lead to the individualization of treatment recommendations.

http://thejns.org/doi/abs/10.3171/2015.1.JNS14681

KEY WORDS obsessive-compulsive disorder; cingulotomy; capsulotomy; stereotactic lesions; psychiatric neurosurgery; functional neurosurgery

$\mathrm{O}$ BSESSIVE-compulsive disorder (OCD) is characterized by repetitive and intrusive thoughts and behaviors that cause clinically significant distress or impairment. ${ }^{2}$ The estimated prevalence of OCD in the US is $2.3 \%$, making it one of the most common psychiatric disorders in the US..$^{27}$ In 2002, the World Health Organization reported that OCD was responsible for nearly $1 \%$ of global years lost due to disability. ${ }^{23}$ Approximately $40 \%$ $60 \%$ of patients with OCD fail to satisfactorily respond to standard treatments, including serotonin reuptake inhibi-

ABBREVIATIONS AE = adverse event; $A H R Q=$ Agency for Healthcare Research and Quality; $C B T C=$ cortico-basal ganglia-thalamocortical; $d A C C=$ dorsal anterior cingulate cortex; DBS = deep brain stimulation; LL = limbic leucotomy; MeSH = Medical Subject Headings; OCD = obsessive-compulsive disorder; OFC = orbitofrontal cortex; PRISMA = Preferred Reporting Items for Systematic reviews and Meta-Analyses; SCT = subcaudate tractotomy; Y-BOCS = Yale-Brown Obsessive Compulsive Scale. SUBMITTED March 24, 2014. ACCEPTED January 20, 2015.

INCLUDE WHEN CITING Published online August 7, 2015; DOI: 10.3171/2015.1.JNS14681.

DISCLOSURE The authors report no conflict of interest concerning the materials or methods used in this study or the findings specified in this paper. 
tors and cognitive behavioral therapy. These patients are potential candidates for neurosurgical intervention.

The advent of stereotaxy in the mid-20th century led to the development of precise and reproducible lesion procedures for psychiatric indications, including dorsal anterior cingulotomy and anterior capsulotomy. ${ }^{3,18,22}$ The mechanism of action for both of these procedures is typically framed in relation to aberrancies in the affective corticobasal ganglia-thalamocortical (CBTC) circuit. ${ }^{1,5}$ Dorsal anterior cingulotomy, a lesion in the dorsal anterior cingulate cortex (dACC) and cingulum bundle, disrupts bidirectional signaling between the $\mathrm{dACC}$ and the orbitofrontal cortex (OFC), ventral striatum, and limbic structures. Anterior capsulotomy, which targets the anterior limb of the internal capsule, is thought to disrupt communication among the OFC, dACC, ventral striatum, and thalamus.

Independent bodies of evidence support the efficacy of cingulotomy and capsulotomy in the management of treatment-refractory OCD. However, we are aware of only 2 studies that directly compared the 2 procedures, and the most recent was conducted in 1982.9,17 Given the potential benefit of neuromodulatory procedures for intractable psychiatric and neurological disorders, it is critical to understand the evidence supporting these procedures, as well as their adverse effect profiles.

The primary objective of this study was to evaluate and compare the clinical efficacy and adverse effect profiles of dorsal anterior cingulotomy and anterior capsulotomy for the treatment of severe, refractory OCD. This systematic review was conducted in compliance with the Preferred Reporting Items for Systematic reviews and Meta-Analyses (PRISMA) ${ }^{24}$ as well as the Agency for Healthcare Research and Quality (AHRQ) recommendations (www.effectivehealthcare.ahrq.gov) for comparative effectiveness reviews, where appropriate.

\section{Methods}

\section{Literature Search Strategy and Data Sources}

The following electronic databases were searched for primary studies through October 2013: MEDLINE, PubMed, PsycINFO, Scopus, and Web of Knowledge. The search strategy used index terms, such as Medical Subject Headings (MeSH), and key words, as applicable. There were no language restrictions. Conference proceedings were included. Table 1 provides a representative example of the database search strategy implemented in MEDLINE.

In an effort to reduce publication bias, gray literature (for example, unpublished data) was obtained by searching clinical trial registries including ClinicalTrials.gov, National Research Register, and metaRegister of Controlled Trials. Additional information was gathered by hand searching bibliographies from selected papers as well as collections of articles known to the study authors.

\section{Eligibility Criteria \\ Study Selection}

The search results were compiled, and duplicate citations were deleted. One reviewer assessed the titles and abstracts of these studies for potential relevance. Full text articles were identified for the potentially relevant citations. These articles were examined, and study eligibility was determined in an unblinded fashion. Only papers with the most current follow-up data for each group of investigators were included. Case studies were excluded from review. All other study designs were considered for inclusion. Selection criteria are summarized in Table 2.

\section{Participants}

The target study population constituted adults (age $\geq 18$ years old) with severe, refractory OCD and no history of surgery for a psychiatric indication. We excluded studies with patients whose history included psychiatric neurosurgery to reduce the risk of attributing clinical outcome to the cumulative effect of multiple surgeries. However, many of the studies meeting all other selection criteria included results from 1 or more patients who had undergone repeat surgery. Fortunately, many of these studies provided individual patient results, allowing for the exclusion of participants who had undergone more than 1 procedure. Individual participants were included if both of the following criteria were met: 1) the second procedure was a reoperation of the same type as the first (for example, cingulotomy followed by cingulotomy was included, whereas cingulotomy followed by subcaudate tractotomy was excluded); and 2) reoperation took place within a few months of the initial procedure because of the insufficiency of the first procedure, as indicated by postoperative neuroimaging or clinical assessment.

Studies that did not provide sufficient detail to exclude individual participants were selected if they met the following conditions: 1) less than a quarter of the participants underwent a second procedure; 2) the second procedure was a reoperation of the same type as the first (as explained above); and 3) reoperation took place within a few months of the initial procedure because of the insufficiency of the first procedure, as indicated by postoperative neuroimaging or clinical assessment.

\section{Interventions}

Bilateral cingulotomy and capsulotomy for the primary indication of OCD were the exclusive interventions of interest. Surgical and radiosurgical techniques were included. Stereotactic guidance with MRI was required for inclusion as this technique is most relevant to current practice. Studies that used other methods (that is, CT only or ventriculography) were excluded. Variations in lesion technique with regard to lesion location or radiation dose were noted, although these did not influence study eligibility. Studies comparing the interventions to each other or to placebo, as well as noncomparative studies, were considered for inclusion. Studies combining either procedure of interest with an adjunct lesion procedure were excluded (for example, limbic leucotomy).

\section{Outcomes}

The primary outcome was clinical improvement of OCD symptoms, as measured by a change in the YaleBrown Obsessive Compulsive Scale (Y-BOCS) score, ${ }^{11}$ after undergoing either capsulotomy or cingulotomy. Secondary outcomes included changes in depression and 
TABLE 1. Search term combinations for MEDLINE database accessed on October 28, 2013

\begin{tabular}{|c|c|c|c|c|}
\hline \multirow{2}{*}{\multicolumn{2}{|c|}{$\begin{array}{c}\text { Question Components \& Selection } \\
\text { of Relevant Terms }\end{array}$}} & \multicolumn{2}{|c|}{ Type of Term } & \multirow[b]{2}{*}{ Boolean Operator } \\
\hline & & Free & $\mathrm{MeSH}$ & \\
\hline \multicolumn{5}{|c|}{ Population: adults w/ treatment-refractory OCD } \\
\hline 1 & exp Obsessive Compulsive Disorder/ & & $x$ & \multirow[t]{4}{*}{ OR (captures population) } \\
\hline 2 & OCD.mp. & $x$ & & \\
\hline 3 & obsessive compulsive disorder.mp. & $x$ & & \\
\hline 4 & Obsessive-Compulsive Disorder.mp. & $x$ & & \\
\hline 5 & or $(1-4)$ & & & \\
\hline \multicolumn{5}{|c|}{ Interventions: cingulotomy, capsulotomy } \\
\hline 6 & exp Psychosurgeryl & & $x$ & \multirow[t]{6}{*}{ OR (captures intervention) } \\
\hline 7 & exp Stereotaxic Techniques/ & & $x$ & \\
\hline 8 & exp Gyrus Cinguli/ & & $x$ & \\
\hline 9 & cingulotomy.mp. & $x$ & & \\
\hline 10 & capsulotomy.mp. & $x$ & & \\
\hline 11 & anterior capsulotomy.mp. & $x$ & & \\
\hline 12 & or $(6-11)$ & & & \\
\hline \multicolumn{5}{|c|}{ Outcomes } \\
\hline \multicolumn{5}{|c|}{ No search } \\
\hline \multicolumn{5}{|c|}{ Study Designs } \\
\hline \multicolumn{5}{|c|}{ No search } \\
\hline 13 & 5 and 12 & & & AND (combines population and interventions) \\
\hline
\end{tabular}

anxiety rating scale scores and adverse events (AEs), with a separate category for those causing permanent or serious morbidity (for example, hemiplegia, intracranial hemorrhage, seizure disorder, cognitive deficits, personality change, weight gain) or mortality. Studies were excluded for a lack of documentation on primary outcome and for a mean follow-up shorter than 12 months. Depression, anxiety, and AE reporting did not impact study eligibility.

\section{Data Extraction and Data Items}

Data were obtained from eligible studies using a prespecified electronic data collection form. ${ }^{12}$ Collected data included the following: characteristics of study participants, study design and location, definition of treatment-refractory OCD, study eligibility criteria, details of surgical and medical treatment, change in therapeutic regimen during the study period, length of follow-up, method of data collection at each time point, Y-BOCS scores at baseline and available follow-ups, depression and anxiety scores at baseline and subsequent follow-ups, and AEs.

\section{Quality Assessment}

Risk of bias for the primary efficacy outcome was assessed for each individual study using a study design-specific tool developed by the AHRQ. ${ }^{31}$ Assessment of the risk of bias did not play a role in data synthesis.

\section{Synthesis of Results}

The primary outcome was pooled across studies by calculating the weighted mean Y-BOCS score at baseline, 12 months' follow-up, and last follow-up for cingulotomy and capsulotomy groups. The weight was based on the relative proportion of participants from each study that met our inclusion criteria. Adverse event rates were quantified as the percentage of procedures that had complications. Repeat procedures were taken into account. Pooled AEs were calculated using a weighted average within each intervention group. The weight was based on the number of procedures that met inclusion criteria.

\section{Results \\ Study Selection}

A total of 1921 references were retrieved from electronic database searches, gray literature, and hand searches. After excluding 654 duplicates, 1267 references were

TABLE 2. Study selection criteria

\begin{tabular}{l}
\hline Inclusion \\
\hline Adult (age $\geq 18$ yrs) \\
\hline OCD Dx \\
\hline Bilat cingulotomy or bilateral capsulotomy \\
\hline Y-BOCS before \& after intervention \\
\hline Exclusion \\
\hline Case report \\
\hline Previous psychosurgery ${ }^{*}$ \\
\hline Lack of stereotactic MRI guidance \\
\hline Cingulotomy or capsulotomy combined w/ other intervention \\
\hline Mean FU <12 mos \\
\hline Dx = diagnosis; FU = follow-up. \\
${ }^{*}$ See text for exceptions.
\end{tabular}




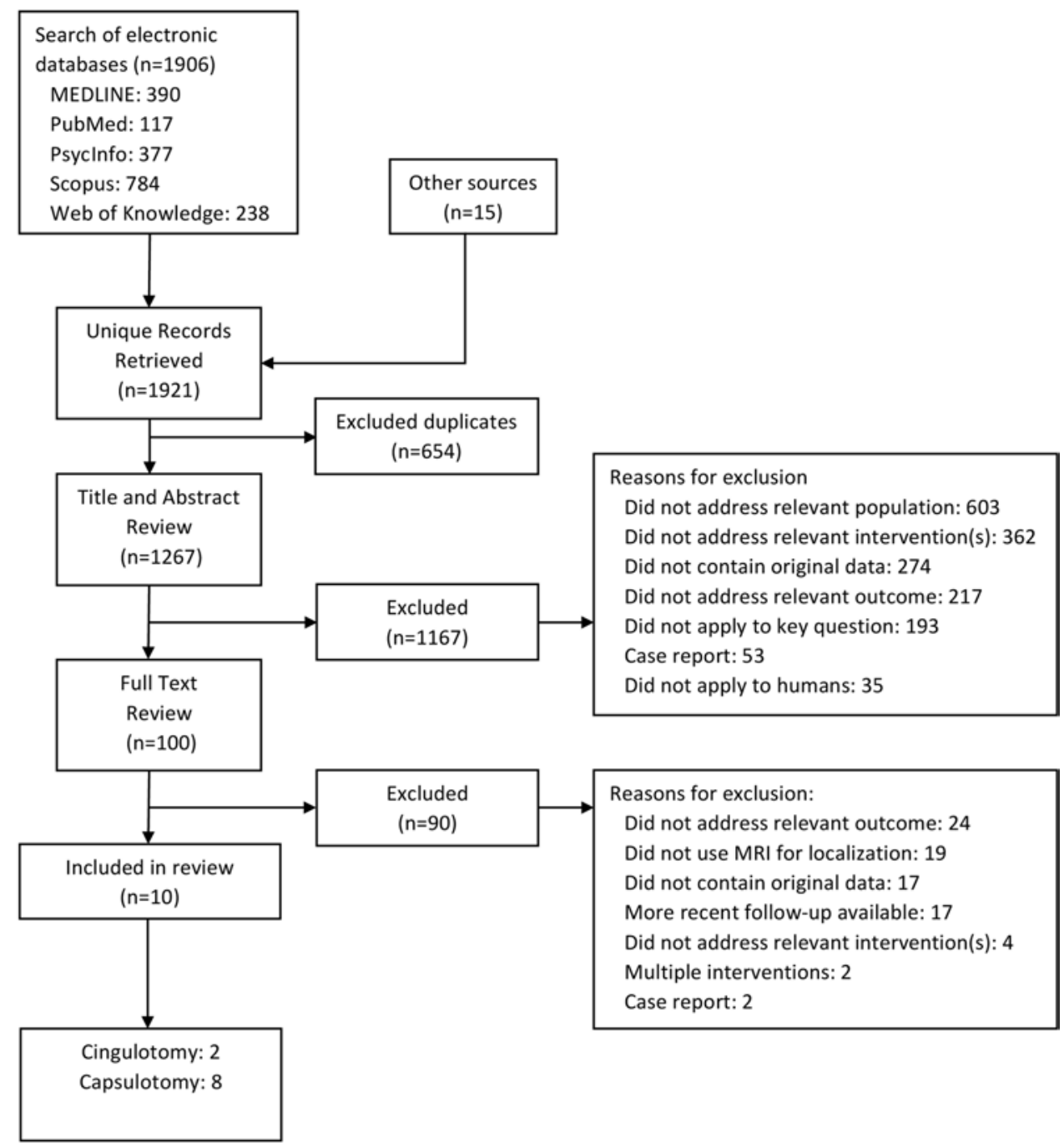

FIG. 1. PRISMA study selection flowchart. The selection process moves from top to bottom, starting with the electronic database search results and ending with the 10 studies included in this review. Exclusions are enumerated at each step in the selection process. Reasons for study exclusion are provided on the right side of the figure.

screened for potential eligibility, of which 1167 were excluded. The remaining 100 references underwent full text review (Fig. 1).

\section{Study Characteristics}

The characteristics of included studies are summarized in Table 3. Two cingulotomy and 8 capsulotomy studies were included in the review.

\section{Study Design}

The majority of included study designs were singlearm prospective cohort observational studies with the following exceptions: 1 retrospective cohort study ${ }^{26}$ and 1 prospective controlled cohort study. ${ }^{6}$

\section{Participants}

All study participants were adults meeting the criteria for OCD in the Diagnostic and Statistical Manual of Mental Disorders. The studies included a total of 193 partici- pants - 81 who underwent cingulotomy and 112 who underwent capsulotomy. Most of the studies required treatment refractoriness as part of the inclusion criteria. ${ }^{6,719,21,25,26,28,29}$ One cingulotomy study ${ }^{14}$ and 4 capsulotomy studies ${ }^{15,19,21,28}$ specified exclusion criteria in the participant selection process. Only 5 studies, all capsulotomy studies, $, 19,21,26,28$ reported on the prevalence of psychiatric comorbidities.

\section{Interventions}

Surgical techniques included both open and radiosurgical methods. Each study reported unique parameters for temperature or radiation dose, number of lesion isocenters, or tracks per side. Rück et al. is notable among the stereotactic radiosurgery capsulotomy studies for using the largest radiation dose and number of isocenters. ${ }^{26}$ Three capsulotomy studies pooled data from patients who had undergone reoperation with those who had undergone a single procedure, $, 19,25$ and 1 study included 1 patient with a history of deep brain stimulation (DBS) for OCD. ${ }^{26}$ The majority of studies did not report co-interventions or ad- 

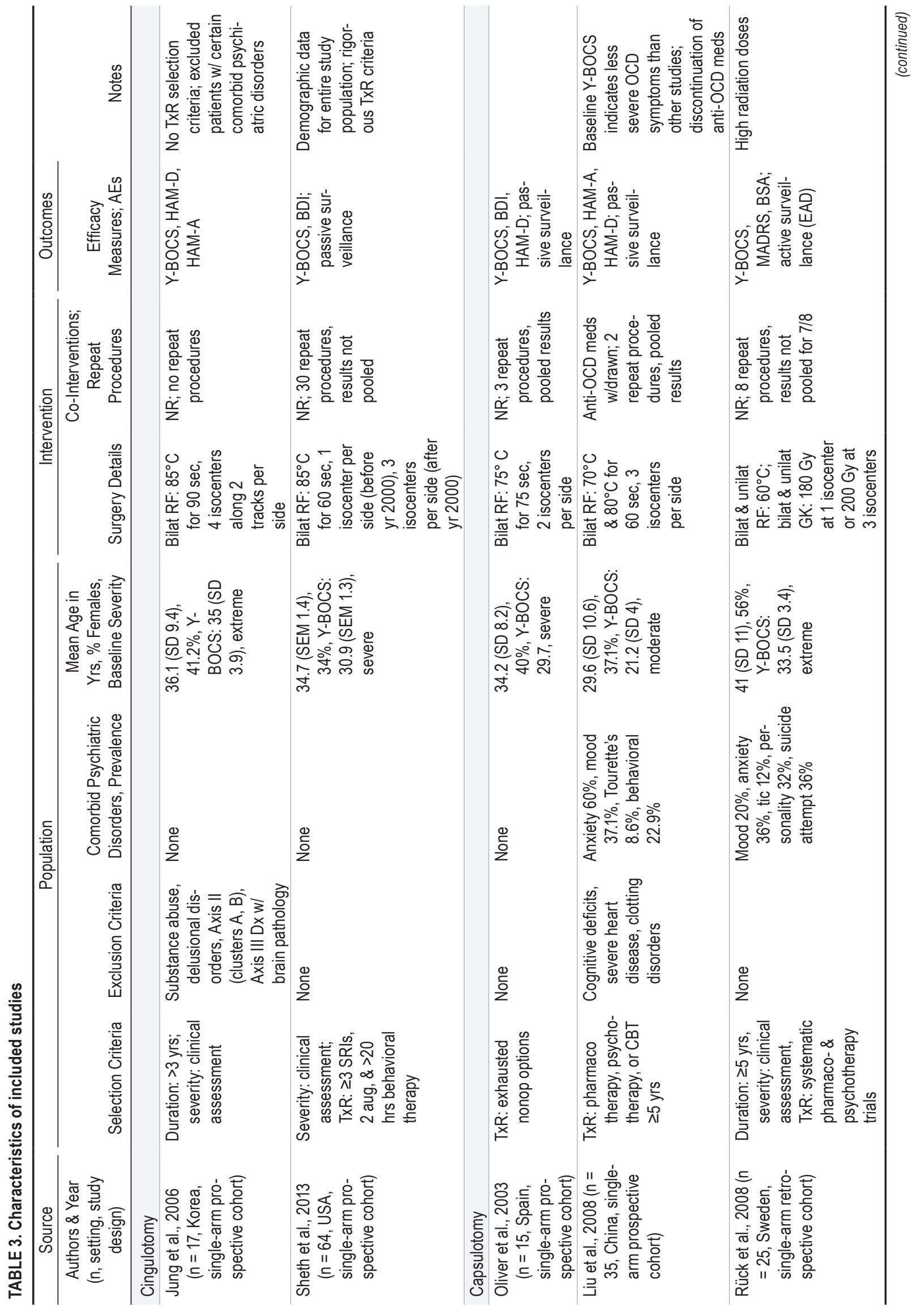


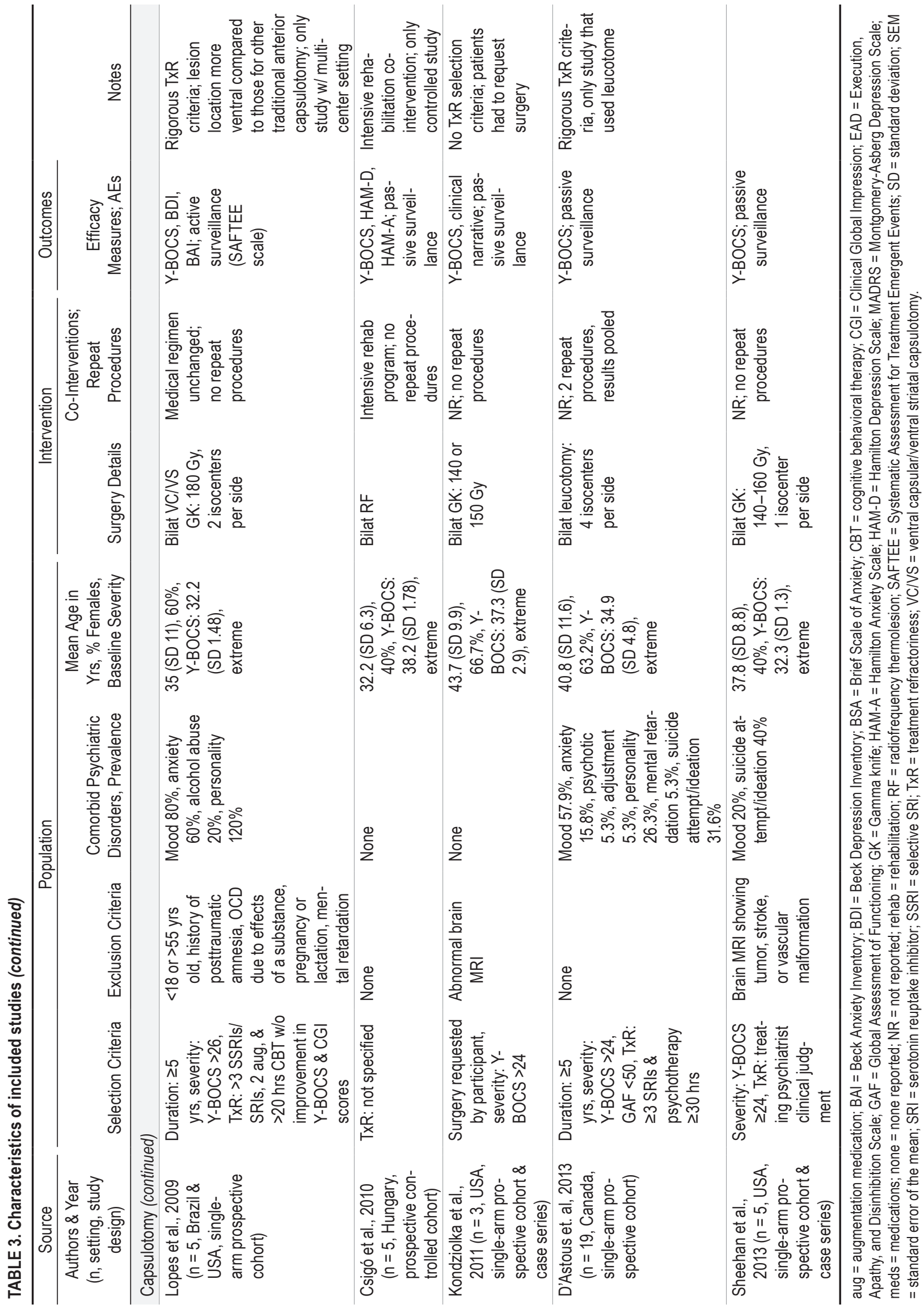


dress potential therapeutic confounders, such as a change in medication regimen at the time of intervention. One study withdrew all anti-OCD medications at the time of capsulotomy, ${ }^{19}$ and another enrolled participants in an intensive rehabilitation program consisting of pharmacoand psychotherapy after surgery. ${ }^{6}$

\section{Outcomes}

Each study quantified OCD symptom severity using the Y-BOCS before and after the procedure and at the long-term follow-up. Nearly all of the studies also provided Y-BOCS data at the 12-month follow-up. ${ }^{6,7,14,19,21,25,26,29}$ Seven studies quantified depression before and after surgery, 6,14,19,21,25,26,29 and 5 studies scored anxiety symp-

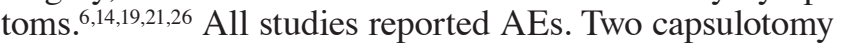
groups employed active surveillance of AEs through the use of a standardized inventory. ${ }^{21,26}$

\section{Quality Assessment}

The assessment of risk of bias for the efficacy outcome is summarized in Table 4.

\section{Individual Study Results}

The Y-BOCS-based efficacy results of the individual studies are summarized in Table 5. Depression and anxiety outcomes are summarized in Table 6. Adverse events for each study are summarized in Table 7.

\section{Synthesis of Results}

Given that the majority of studies were observational and noncomparative, we were unable to perform statistical comparisons between or within cingulotomy and capsulotomy groups. However, individual study results were combined within their respective groups where appropriate.

\section{Characteristics of Participants}

The average age of participants at the time of surgery was $35.3 \pm 10.7$ (mean \pm standard deviation), $35.0 \pm 10.9$, and $35.6 \pm 10.6$ years across all studies, cingulotomy studies, and capsulotomy studies, respectively. The majority of participants were male, comprising $57 \%$ of participants across all studies. The average time to the last follow-up was 55 months (range 22-84 months) for all studies, 47 months (range 24-59 months) for cingulotomy, and 60 months (range 22-84 months) for capsulotomy.

\section{Efficacy}

The Y-BOCS-based efficacy results of individual studies are summarized in Table 5. The mean baseline YBOCS score was 32.3 (range 30.9-35) in the cingulotomy group and 29.3 (range 21.2-38.2) in the capsulotomy group. These scores fall within the extreme and severe ranges, respectively. The mean reduction in the Y-BOCS score at 12 months' follow-up was 37\% (range 36\%-37\%) for cingulotomy and 55\% (range 36\%-75\%) for capsulotomy. At the last follow-up, the mean reduction in the YBOCS score was 37\% (range 31\%-48\%) for cingulotomy and $57 \%$ (range $32 \%-79 \%$ ) for capsulotomy. In keeping with traditional thresholds used in pharmacology trials, full response was defined as a Y-BOCS score reduction $\geq$

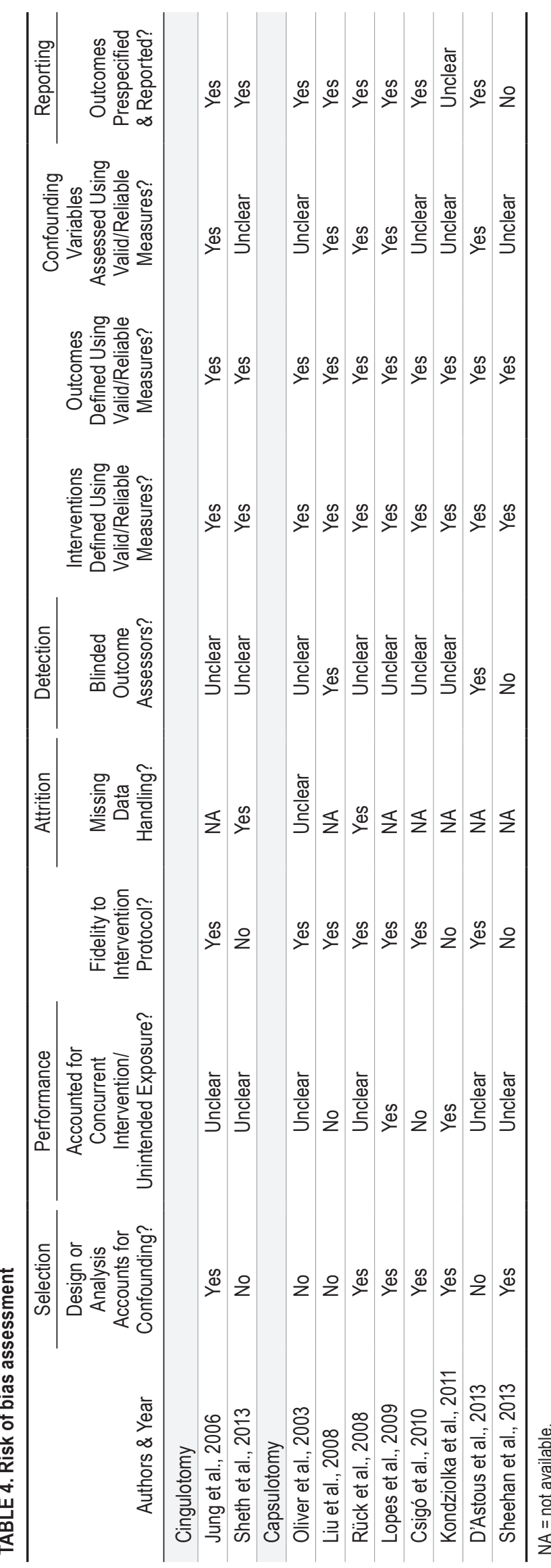

J Neurosurg Volume 124 • January 2016 


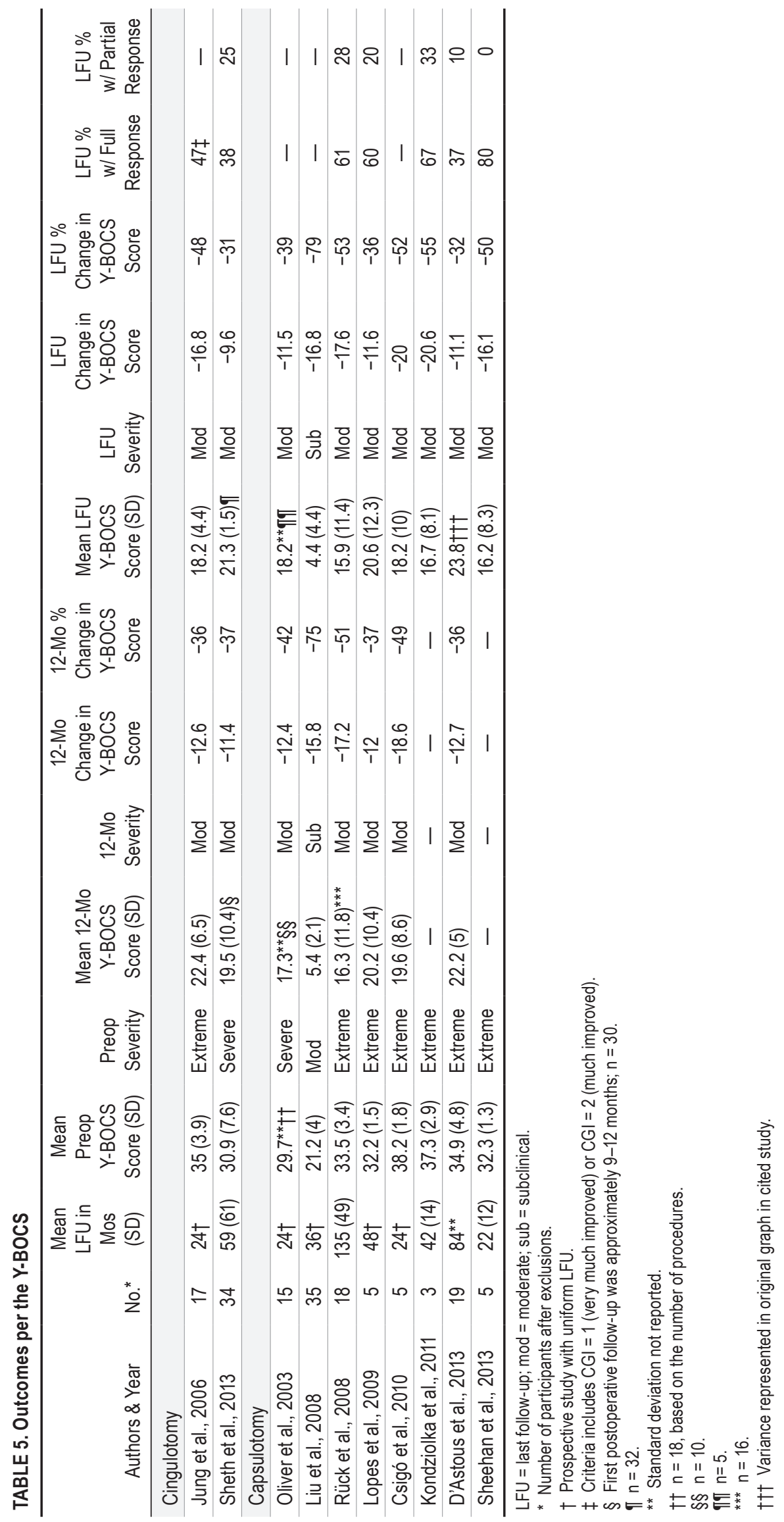


$35 \%$ at the last follow-up, and partial response was defined as a Y-BOCS score reduction $\geq 25 \%$ and $<35 \%$. The mean full response rate for cingulotomy at the last follow-up was $41 \%$ (range $38 \%-47 \%, \mathrm{n}=2$ studies, $\mathrm{n}=51$ participants), and the partial response rate was $25 \%(\mathrm{n}=1$ study, $\mathrm{n}=34$ participants). For capsulotomy, the mean full response rate at the last follow-up was $54 \%$ (range $37 \%-80 \%, \mathrm{n}=5$ studies, $\mathrm{n}=50$ participants) and the partial response rate was $18 \%$ (range $0 \%-33 \%, \mathrm{n}=5$ studies, $\mathrm{n}=50$ participants).

Depression and anxiety outcomes for available studies are presented in Table 6. We were unable to combine results across studies given that the scales used to assess depression and anxiety differed between studies.

\section{Adverse Events}

Adverse events were characterized as the number of events per procedure (Table 7). The rate of transient AEs was $14.3 \%$ (range $13.7 \%-17.6 \%$ ) across cingulotomy studies $(\mathrm{n}=116$ procedures) and $56.2 \%$ (range $0-260 \%$ ) across capsulotomy studies ( $\mathrm{n}=112$ procedures). The rate of serious or permanent AEs was 5.2\% (range 0-6\%) across cingulotomy studies and $21.4 \%$ (range $0-66.7 \%$ ) across capsulotomy studies. It should be noted that the AE rate across cingulotomy studies may be overly elevated as 1 study includes complications from all procedures, including repeat cingulotomy and limbic leucotomy procedures. ${ }^{27}$ In addition, nearly all of the serious or permanent AEs reported by Rück et al. are attributable to 3 patients who had received 200 Gy at 3 isocenters, and thus receiving the greatest radiation exposure of all participants in the reviewed studies. ${ }^{26}$ Excluding this study from the pooled results nearly halves the rate of serious complications in the capsulotomy group to $12.8 \%$ (range $0-40 \%$ ).

\section{Discussion}

\section{Summary of Evidence}

The reviewed literature supports the assertion that dorsal anterior cingulotomy and anterior capsulotomy are effective interventions in the management of severe, refractory OCD. The pooled mean reduction in baseline Y-BOCS score meets the criteria for treatment response following both capsulotomy and cingulotomy at the 12 months' and the long-term follow-ups. In both intervention groups, the Y-BOCS scores appear to change very little between 12 months and the last follow-up, indicating a stable treatment response over time. More than half of the participants who underwent capsulotomy met the criteria for treatment response at the last follow-up (54\%, range $37 \%-80 \%$ ) as well as nearly half of those who underwent cingulotomy $(41 \%$, range $38 \%-47 \%)$. Both procedures carry the risk of AEs. Capsulotomy was associated with 56.2\% transient and/or mild AEs and $21.4 \%$ permanent and/or serious AEs. Excluding Rück et al. from the pooled results yields a $12.8 \%$ serious complication rate for capsulotomy. ${ }^{26}$ Cingulotomy was associated with $14.3 \%$ transient and/or mild AEs and 5.2\% permanent and/or serious AEs. Lastly, both cingulotomy and capsulotomy appear to be efficacious in addressing comorbid depression and anxiety symptoms, as evidenced by a significant reduction in the respective inventory scores following both procedures.

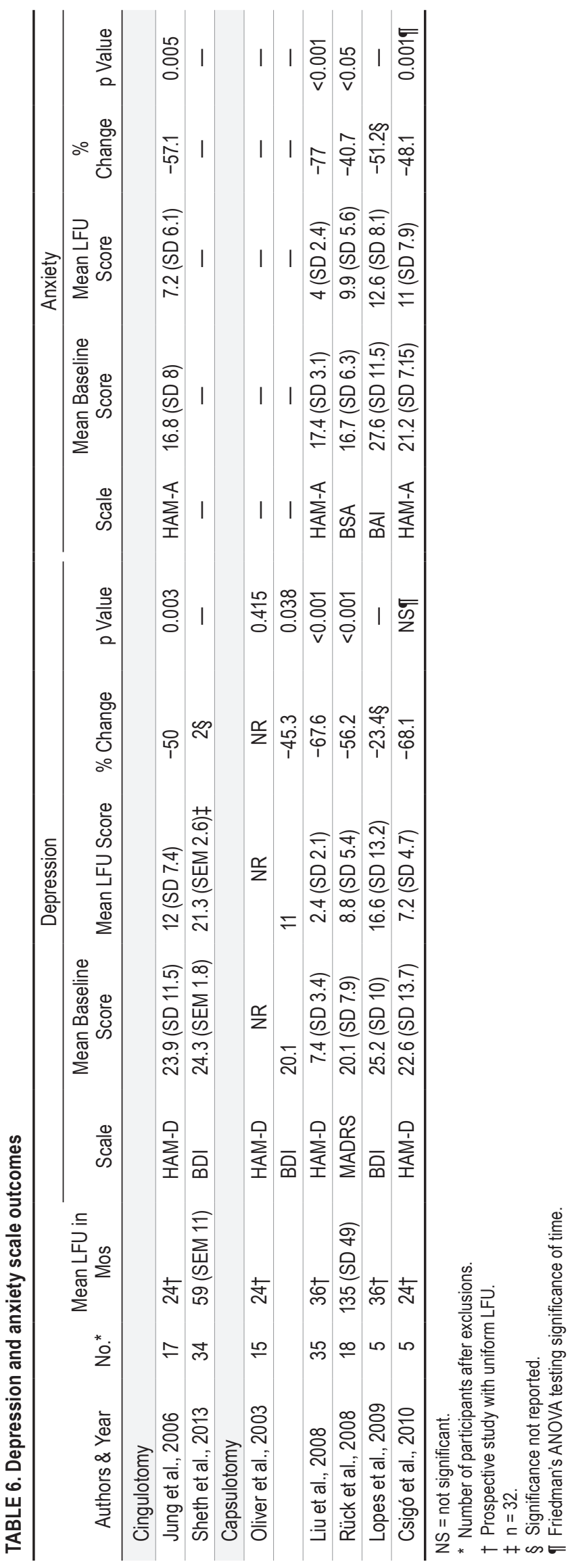




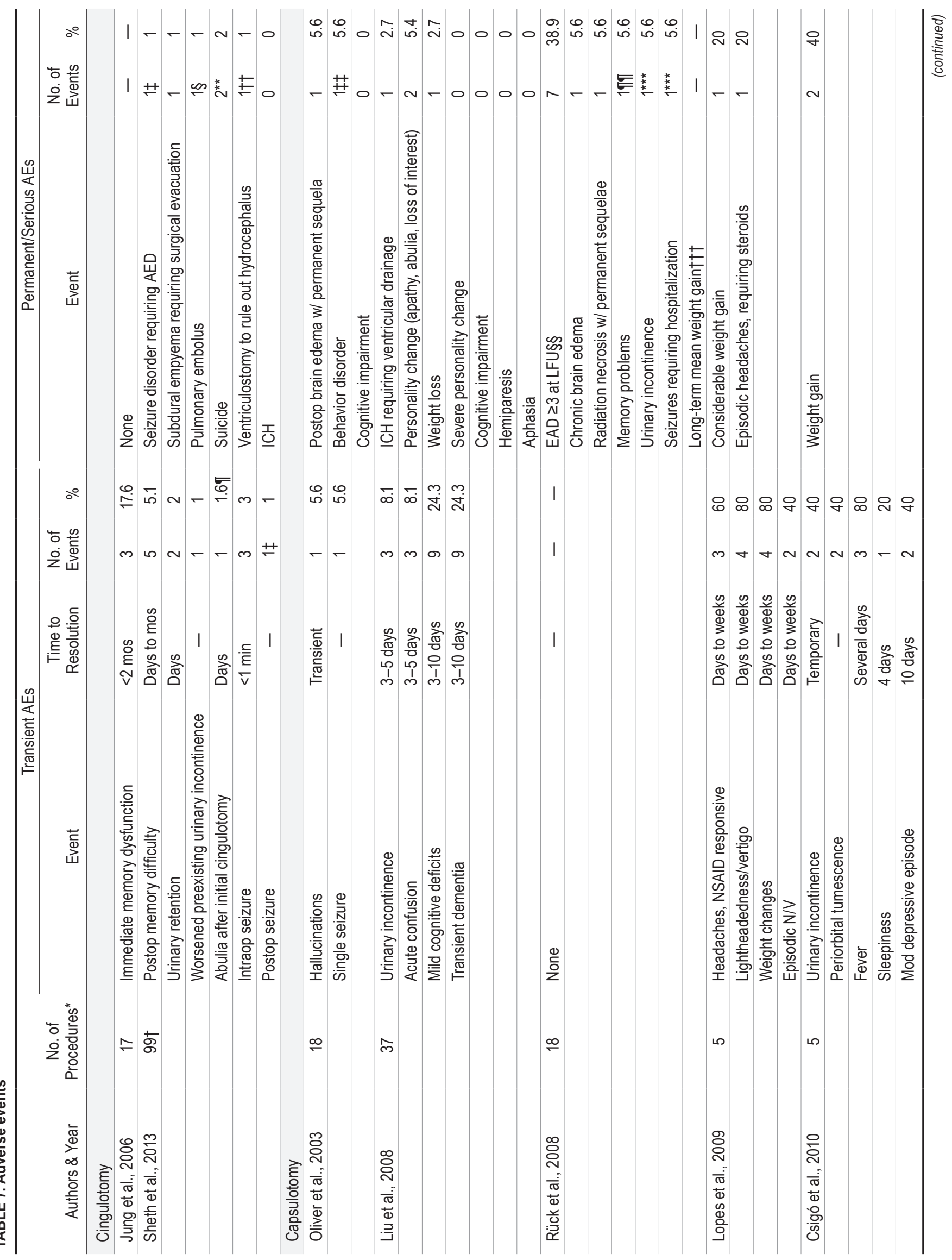




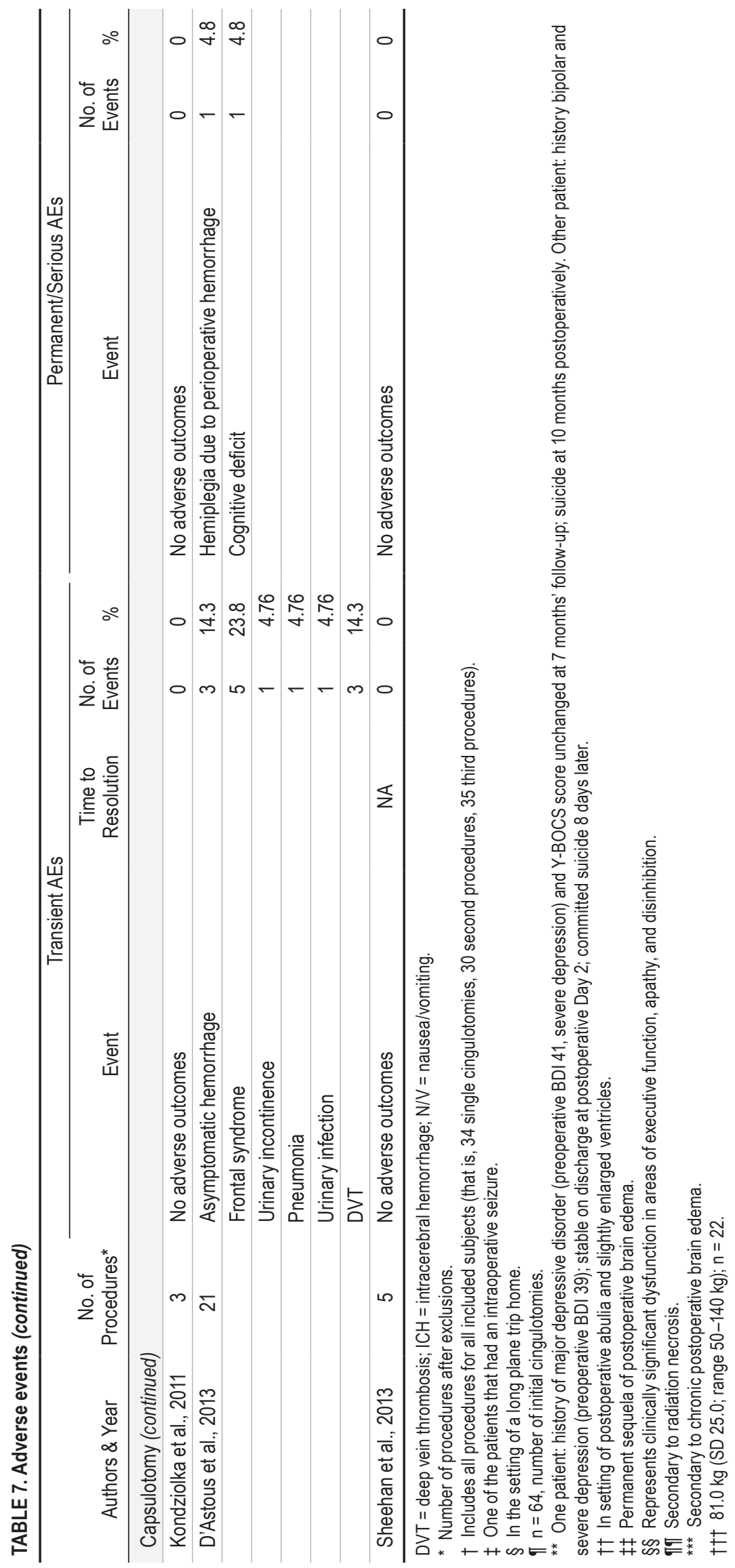




\section{Study Limitations}

Overall, the included studies reflect the population, interventions, and outcomes of interest. Treatment refractoriness and disease severity were important population descriptors for the purposes of this review. Nearly all of the included studies satisfied these 2 criteria. Nevertheless, inconsistent comorbidity reporting across studies makes generalization difficult given the significant impact of psychiatric comorbidity, specifically depression, on quality of life measures in OCD. ${ }^{8,13}$

Interinstitutional heterogeneity in surgical technique was evident in both cingulotomy and capsulotomy studies. Variation in radiation dosage, number of radiosurgical isocenters, thermolesion temperature dosage, and lesion location must be taken into account when generalizing to current neurosurgical practice. This heterogeneity is of particular relevance to AEs. Rück et al. illustrate an association between excessive radiation exposure and risk of permanent AEs. ${ }^{26}$ In their report, the authors conceded that the dose was too high and probably accounted for the complications observed in those patients. Removing this outlier study from our analysis greatly reduced the AE rate for capsulotomy, thereby highlighting the need for careful consideration of individual technique and event reporting before casting broad generalizations on the safety of either capsulotomy or cingulotomy. Active surveillance of AEs in future studies would facilitate comparison within and across intervention groups.

All included studies used the Y-BOCS to assess symptom severity prior to surgery and at follow-up. The validity and reliability of the Y-BOCS for measuring OCD symptom severity has been well established; however, the relationship between Y-BCOS scores and quality of life measures is less well characterized. A number of studies have found that OCD symptoms have a significant effect on quality of life, but this relationship is not as well established as that between depressive symptoms and quality of life..$^{10,13,16,30}$ Fortunately, the reviewed literature supports the role of cingulotomy and capsulotomy in treating comorbid depressive symptoms as well.

A major limitation of this study is its composition of solely observational studies without controls. The nature of these study designs increases the risk of bias due to compromised internal validity (Table 4). Furthermore, the lack of comparison in the designs of the included studies does not support the direct or indirect comparison of outcomes between cingulotomy and capsulotomy. Controlled trials are necessary to determine the relative efficacy between the 2 procedures. The results of this systematic review must be interpreted within the context of the strengths and weaknesses of the included studies.

Currently, the choice of which lesion procedure to offer is largely based on historic institutional practice. As highlighted in this systematic review, no data support the application of one procedure over the other in terms of efficacy or safety profile. Future studies should strive for homogeneity of technique and careful documentation of OCD subtype and neuropsychological profile. Head-to-head comparisons, even in a blinded fashion potentially, would be ethically feasible given current clinical equipoise. Because the procedures target different regions of the same CBTC circuit, it is quite possible that such comparisons would reveal subtle differences in response, allowing tailoring of recommendations based on individual symptoms.

We did not include DBS studies in this systematic review for a number of reasons. First, a recent article has thoroughly reviewed the literature of DBS for OCD. ${ }^{4}$ Whereas that article is not a "systematic review," we believe that the information presented in our current paper can be easily compared with the information presented in that article and that further recapitulation of the same information would be redundant. Second, there is significant heterogeneity in the DBS literature (summarized in Blomstedt et al. ${ }^{4}$ ) in terms of study design and reporting. Given the limitations mentioned above within just the lesion literature, we believe that inclusion of the DBS literature would further limit the utility of a systematic review. Third, DBS has been available for a comparably shorter period of time; therefore, the duration of follow-up is less than that for lesions. For example, the last follow-up intervals in the lesion studies included in the present review ranged from 22 to 135 months, whereas those in some of the DBS studies were as short as 3 months.

We also chose not to include subcaudate tractotomy (SCT) and limbic leucotomy (LL) in this systematic review. A dearth of studies report OCD outcomes for SCT and LL in the literature. Search protocols similar to the ones used for cingulotomy and capsulotomy were used to query PubMed for articles published within the past 10 years that reported LL or SCT outcomes for OCD. The initial search yielded 21 articles for SCT and 34 articles for LL, published since January 1, 2003. After applying our study inclusion criteria, only 1 of the articles covering SCT or LL would have been included. Therefore, SCT and LL were not included in the current systematic review.

Despite the limitations of this study, cingulotomy and capsulotomy remain important parts of the neurosurgical armamentarium for the treatment of severe, refractory OCD. These procedures are quite relevant in contemporary practice, as evidenced by the fact that 3 of the 10 studies were published in 2013. Lopes and colleagues recently published the results of a randomized controlled trial of gamma ventral capsulotomy for OCD, the first such study to evaluate lesion outcomes for OCD. ${ }^{20}$ This study further supports the modern relevance of lesion studies as well as the feasibility of employing a randomized blinded study design to measure clinical outcomes. With the advent of newer methods of lesioning (laser ablation, focused ultrasound), it is likely that stereotactic lesions will continue to play an important role in functional neurosurgery.

\section{Conclusions}

The available clinical evidence supports the efficacy of both cingulotomy and capsulotomy in treating severe, refractory OCD, as well as comorbid depressive and anxiety symptoms. Current evidence is insufficient to directly compare cingulotomy and capsulotomy, and recommendations on when to choose one procedure over the other cannot be made. Active AE surveillance is necessary to compare negative outcomes between the 2 interventions. Future controlled comparative studies are necessary to 
accurately compare responses to cingulotomy and capsulotomy and may shed light on subtle differences in patient response that can be used to provide individualized treatment recommendations.

\section{References}

1. Alexander GE, DeLong MR, Strick PL: Parallel organization of functionally segregated circuits linking basal ganglia and cortex. Annu Rev Neurosci 9:357-381, 1986

2. American Psychiatric Association: Diagnostic and Statistical Manual of Mental Disorders: DSM-5. Washington, DC: American Psychiatric Association, 2013

3. Ballantine HT Jr, Cassidy WL, Flanagan NB, Marino R Jr: Stereotaxic anterior cingulotomy for neuropsychiatric illness and intractable pain. J Neurosurg 26:488-495, 1967

4. Blomstedt P, Sjöberg RL, Hansson M, Bodlund O, Hariz MI: Deep brain stimulation in the treatment of obsessive-compulsive disorder. World Neurosurg 80:e245-e253, 2013

5. Bourne SK, Eckhardt CA, Sheth SA, Eskandar EN: Mechanisms of deep brain stimulation for obsessive compulsive disorder: effects upon cells and circuits. Front Integr Neurosci 6:29, 2012

6. Csigó K, Harsányi A, Demeter G, Rajkai C, Németh A, Racsmány M: Long-term follow-up of patients with obsessivecompulsive disorder treated by anterior capsulotomy: a neuropsychological study. J Affect Disord 126:198-205, 2010

7. D'Astous M, Cottin S, Roy M, Picard C, Cantin L: Bilateral stereotactic anterior capsulotomy for obsessive-compulsive disorder: long-term follow-up. J Neurol Neurosurg Psychiatry 84:1208-1213, 2013

8. Eisen JL, Mancebo MA, Pinto A, Coles ME, Pagano ME, Stout R, et al: Impact of obsessive-compulsive disorder on quality of life. Compr Psychiatry 47:270-275, 2006

9. Fodstad H, Strandman E, Karlsson B, West KA: Treatment of chronic obsessive compulsive states with stereotactic anterior capsulotomy or cingulotomy. Acta Neurochir (Wien) 62:1-23, 1982

10. Fontenelle IS, Fontenelle LF, Borges MC, Prazeres AM, Rangé BP, Mendlowicz MV, et al: Quality of life and symptom dimensions of patients with obsessive-compulsive disorder. Psychiatry Res 179:198-203, 2010

11. Goodman WK, Price LH, Rasmussen SA, Mazure C, Fleischmann RL, Hill CL, et al: The Yale-Brown Obsessive Compulsive Scale. I. Development, use, and reliability. Arch Gen Psychiatry 46:1006-1011, 1989

12. Higgins JP, Deeks JJ (eds): Chapter 7: Selecting studies and collecting data. Cochrane Handbook for Systematic Reviews of Interventions, ed 5.1.0. (http://www.cochranehandbook.org) [Accessed May 20, 2015]

13. Huppert JD, Simpson HB, Nissenson KJ, Liebowitz MR, Foa EB: Quality of life and functional impairment in obsessivecompulsive disorder: a comparison of patients with and without comorbidity, patients in remission, and healthy controls. Depress Anxiety 26:39-45, 2009

14. Jung HH, Kim CH, Chang JH, Park YG, Chung SS, Chang JW: Bilateral anterior cingulotomy for refractory obsessivecompulsive disorder: Long-term follow-up results. Stereotact Funct Neurosurg 84:184-189, 2006

15. Kondziolka D, Flickinger JC, Hudak R: Results following gamma knife radiosurgical anterior capsulotomies for obsessive compulsive disorder. Neurosurgery 68:28-32, 23, 2011

16. Kugler BB, Lewin AB, Phares V, Geffken GR, Murphy TK, Storch EA: Quality of life in obsessive-compulsive disorder: the role of mediating variables. Psychiatry Res 206:43-49, 2013

17. Kullberg G: Differences in effect of capsulotomy and cingulotomy, in Sweet WH, Brador S, Martin-Rodriguez JG (eds):
Neurosurgical Treatment in Psychiatry, Pain and Epilepsy. Baltimore: University Park Press, 1977, pp 208-301

18. Leksell L: A stereotaxic apparatus for intracerebral surgery. Acta Chir Scand 99:229-233, 1950

19. Liu K, Zhang H, Liu C, Guan Y, Lang L, Cheng Y, et al: Stereotactic treatment of refractory obsessive compulsive disorder by bilateral capsulotomy with 3 years follow-up. J Clin Neurosci 15:622-629, 2008

20. Lopes AC, Greenberg BD, Canteras MM, Batistuzzo MC, Hoexter MQ, Gentil AF, et al: Gamma ventral capsulotomy for obsessive-compulsive disorder: a randomized clinical trial. JAMA Psychiatry 71:1066-1076, 2014

21. Lopes AC, Greenberg BD, Norén G, Canteras MM, Busatto GF, de Mathis ME, et al: Treatment of resistant obsessivecompulsive disorder with ventral capsular/ventral striatal gamma capsulotomy: a pilot prospective study. J Neuropsychiatry Clin Neurosci 21:381-392, 2009

22. Mashour GA, Walker EE, Martuza RL: Psychosurgery: past, present, and future. Brain Res Brain Res Rev 48:409-419, 2005

23. Mathers CD, Stein C, Ma Fat D, Rao C, Inoue M, Tomijima N, et al: Global Burden of Disease 2000: Version 2 methods and results. (http://www.who.int/healthinfo/paper50. pdf) [Accessed May 20, 2015]

24. Moher D, Liberati A, Tetzlaff J, Altman DG: Preferred reporting items for systematic reviews and meta-analyses: the PRISMA statement. BMJ 339:b2535, 2009

25. Oliver B, Gascón J, Aparicio A, Ayats E, Rodriguez R, Maestro De León JL, et al: Bilateral anterior capsulotomy for refractory obsessive-compulsive disorders. Stereotact Funct Neurosurg 81:90-95, 2003

26. Rück C, Karlsson A, Steele JD, Edman G, Meyerson BA, Ericson K, et al: Capsulotomy for obsessive-compulsive disorder: long-term follow-up of 25 patients. Arch Gen Psychiatry 65:914-921, 2008

27. Ruscio AM, Stein DJ, Chiu WT, Kessler RC: The epidemiology of obsessive-compulsive disorder in the National Comorbidity Survey Replication. Mol Psychiatry 15:53-63, 2010

28. Sheehan JP, Patterson G, Schlesinger D, Xu Z: Gamma knife surgery anterior capsulotomy for severe and refractory obsessive-compulsive disorder. J Neurosurg 119:1112-1118, 2013

29. Sheth SA, Neal J, Tangherlini F, Mian MK, Gentil A, Cosgrove GR, et al: Limbic system surgery for treatment-refractory obsessive-compulsive disorder: a prospective long-term follow-up of 64 patients. J Neurosurg 118:491-497, 2013

30. Subramaniam M, Soh P, Vaingankar JA, Picco L, Chong SA: Quality of life in obsessive-compulsive disorder: impact of the disorder and of treatment. CNS Drugs 27:367-383, 2013

31. Viswanathan M, Ansari M, Berkman N, Hartling L, McPheeters M, Santaguida PL, et al: Assessing the risk of bias of individual studies in systematic reviews of health care interventions, in Methods Guide for Comparative Effectiveness Reviews. Rockville, MD: Agency for Healthcare Research and Quality, 2012

\section{Author Contributions}

Conception and design: Sheth, Brown, Mikell, Youngerman. Acquisition of data: Brown. Analysis and interpretation of data: Sheth, Brown, Mikell, Youngerman, Zhang. Drafting the article: Sheth, Brown, Mikell. Critically revising the article: all authors. Reviewed submitted version of manuscript: all authors. Statistical analysis: Brown, Zhang. Study supervision: Sheth, Mikell.

\section{Correspondence}

Sameer A. Sheth, Department of Neurological Surgery, The Neurological Institute, NI-551, 710 W. 168th St., New York, NY 10032. email: ss4451@columbia.edu. 